\title{
ON SPECTRAL ESTIMATION
}

BY C. A. SWANSON

Communicated by R. P. Boas, September 14, 1961

Inequalities involving linear transformations on Hilbert space and their spectral families will be derived for the purpose of obtaining information about the location of the spectrum. The theorems obtained are extensions of those given by the author [6] and H. D. Block and W. H. J. Fuchs [1] to unbounded transformations for which a spectral decomposition theorem is valid. Such transformations include maximal normal and self-adjoint as well as bounded symmetric and unitary transformations. The domain $\mathfrak{D}_{T}$ of every linear transformation $T$ being considered is supposed to be dense in Hilbert space $\mathfrak{S}$.

THEOREM 1. Let $T$ be a self-adjoint linear transformation and let $E(\lambda)$ be the associated spectral family of projections. For an arbitrary real number $\alpha$ and an arbitrary positive number $\epsilon$, let $\Lambda$ denote the open interval $(\alpha-\epsilon, \alpha+\epsilon)$ and let $P(\Lambda)$ denote the projection $E(\alpha+\epsilon-0)$ $-E(\alpha-\epsilon)$. Then for every $x \in \mathfrak{D}_{T}$ and $v \in E(\alpha-0) \mathfrak{D}_{T}$, the following inequalities are valid:

$$
\begin{aligned}
\|T x-\alpha x\| & \geqq \epsilon\|x-P(\Lambda) x\| ; \\
(\alpha v-T v, v) & \geqq \epsilon\|v-P(\Lambda) v\|^{2} .
\end{aligned}
$$

Proof. According to the spectral decomposition theorem for selfadjoint transformations [5, p. 180],

$$
\begin{aligned}
\|T x-\alpha x\|^{2} & =\int_{-\infty}^{\infty}|\lambda-\alpha|^{2} d\|E(\lambda) x\|^{2} \geqq \epsilon^{2} \int_{|\lambda-\alpha| \geq \epsilon} d\|E(\lambda) x\|^{2} \\
& =\epsilon^{2}\|x-P(\Lambda) x\|^{2} .
\end{aligned}
$$

This proves (1). To prove (2), let $v=E(\alpha-0) x$ where $x \in \mathfrak{D}_{T}$. Then $E(\alpha-\epsilon) v=E(\alpha+\epsilon-0) v-P(\Lambda) v=v-P(\Lambda) v$, and the spectral decomposition theorem gives

$$
\begin{aligned}
(\alpha v-T v, v) & =\int_{-\infty}^{\alpha}(\alpha-\lambda) d(E(\lambda) v, v) \geqq \epsilon \int_{-\infty}^{\alpha-\epsilon} d(E(\lambda) v, v) \\
& =\epsilon(E(\alpha-\epsilon) v, v)=\epsilon(v-P(\Lambda) v, v) .
\end{aligned}
$$

The first inequality of Theorem 1 generalizes without difficulty to normal transformations $[4$, p. $355 ; 5, \mathrm{pp} .311-331]$. The proof follows Theorem 1 and will be omitted. 
TheOREM 2. Let $T$ be a maximal normal linear transformation. For an arbitrary complex number $\alpha$ and an arbitrary positive number $\epsilon$, let $\Lambda$ denote the open disk $|\lambda-\alpha|<\epsilon$, and let $P(\Lambda)$ denote the associated spectral measure for $T$. Then the inequality

$$
\|T x-\alpha x\| \geqq \epsilon\|x-P(\Lambda) x\|
$$

is valid for all $x \in \mathfrak{D}_{\text {T. }}$.

As a first application of these inequalities, we shall obtain some enclosure theorems of the type given by Block and Fuchs [1] for matrices. Let $S$ denote the spectrum of $T$. Suppose that $\alpha$ is an arbitrary number, in general not in $S$, and suppose $\epsilon$ is selected to be $\min _{\lambda \in S}|\lambda-\alpha|$. The minimum is attained since $S$ is a closed set in the complex plane. Then $\Lambda \cap S$ is empty and hence $P(\Lambda)=0$ [3]. It is a consequence of (3) that for any $x \in \mathfrak{D}_{T}$,

$$
\min _{\lambda \in S}|\lambda-\alpha| \leqq\|T x-\alpha x\| /\|x\| \text {. }
$$

In the case that $T$ is self-adjoint, (1) is used instead of (3) and (2) also can be used to yield

$$
\min _{\lambda \in S}|\lambda-\alpha| \leqq(\alpha v-T v, v) /\|v\|^{2},
$$

for $v=E(\alpha-0) x, x \in \mathscr{D}_{T}$. The notations $\rho(T, \alpha ; x)$ and $\sigma(T, \alpha ; x)$ will be used for the right members of (4) and (5). The results are summarized in the following theorems.

TheOREM 3. Under the assumptions of Theorem 2, the intersection of the disk $|\lambda-\alpha| \leqq \rho(T, \alpha ; x), x \in \mathfrak{D}_{\boldsymbol{T}}$ with the spectrum of $T$ is nonempty.

THEOREM 4. Under the assumptions of Theorem 1, the intersection of the interval $[\alpha-\sigma(T, \alpha ; x), \alpha]$ with the spectrum of $T$ is nonempty.

Both theorems are applicable in the case of a symmetric, completely continuous transformation on $\mathfrak{S}$. For the purpose of locating an eigenvalue $\lambda_{m}\left(\lambda_{m}<\lambda_{m-1} \leqq \cdots\right)$, the choice $\alpha=(T x, x)$ is made, and it turns out that the estimate of Theorem 4 is asymptotically sharper than that of Theorem 3. This means that there exists a positive number $\eta$ such that

$$
\sigma(T,(T x, x) ; x)<\rho(T,(T x, x) ; x)
$$

for all $x \in \mathfrak{S}$ satisfying $\left\|x-y_{m}\right\| \leqq \eta(m=1,2, \cdots)$. The proof consists of some computations with the aid of the Parseval formula and the Schwarz inequality, and will be omitted. 
In certain aspects of perturbation theory [2], $\alpha$ is chosen to be an isolated point in the spectrum of a transformation $A$ which is $\delta$-close to $T(\delta>0)$ in the sense that there exists a nonempty (characteristic) subspace $\mathfrak{U} \subset \mathscr{D}_{T}$ such that

$$
\|T x-\alpha x\| \leqq \delta\|x\| \quad \text { for all } x \in \mathfrak{A} .
$$

The positive number $\delta$ is a measure of the closeness of $\alpha$ to $T$. The following theorems give estimates for the closeness of $\alpha$ to the spectrum of $T$.

TheOREM 5. Let $T$ be maximal normal and let $\alpha$ be $\delta$-close to $T$. If $\Delta$ denotes the closed disk $|\lambda-\alpha| \leqq \delta$, then $\operatorname{dim}[P(\Delta) \mathfrak{A}] \geqq \operatorname{dim} \mathfrak{A}$.

TheOREM 6. Let $T$ be symmetric and completely continuous on $\mathfrak{S}$. Let $\mathfrak{A}$ be an m-dimensional subspace of $\mathfrak{S}$, let $\alpha$ be real with $\alpha \geqq\|T\|$ and let $\delta$ be positive. If $(\alpha x-T x, x) \leqq \delta(x, x)$ for all $x \in \mathfrak{A}$, then at least $m$ eigenvalues of $T$ lie in the interval $[\alpha-\delta, \alpha]$.

The type of perturbation described above arises in connection with differential operators when the perturbation arises (nonanalytically) from a change of domain of the operator. In such applications, property (7) is established in a preliminary step by using special properties of differential equations such as the maximum principle for elliptic equations.

To prove Theorem 5 , we combine (3) and (7) to obtain $\|x-P(\Lambda) x\|$ $\leqq(\delta / \epsilon)\|x\|, x \in \mathfrak{A}$. Let $\theta$ be an arbitrary number satisfying $0<\theta<1$ and choose $\epsilon$ to be $\delta / \theta$. Then $\Lambda$ is the disk $|\lambda-\alpha|<\delta / \theta$ and $\|x-P(\Lambda) x\|$ $\leqq \theta\|x\|<\|x\|$. Hence $P(\Lambda) x=0$ implies $x=0$, and $\operatorname{dim}[P(\Lambda) \mathfrak{A}] \geqq \operatorname{dim} \mathfrak{A}$. Since $\theta$ is arbitrary, this proves Theorem 5 . The proof of Theorem 6 is similar and will be omitted.

\section{REFERENCES}

1. H. D. Block and W. H. J. Fuchs, An enclosure theorem for eigenvalues, Bull. Amer. Math. Soc. vol. 67 (1961) pp. 425-426.

2. H. F. Bohnenblust, C. R. DePrima and C. A. Swanson, Elliptic operators with perturbed domains, to appear.

3. P. R. Halmos, Introduction to Hilbert space and the theory of spectral multiplicity, New York, Chelsea, 1951. 1955.

4. F. Riesz and B. Sz.-Nagy, Functional analysis, New York, Frederick Ungar,

5. M. H. Stone, Linear transformations in Hilbert space, Amer. Math. Soc. Colloq. Publ. vol. 15, Amer. Math. Soc., New York, 1932. Reprinted 1951.

6. C. A. Swanson, An inequality for linear transformations with eigenvalues, Bull. Amer. Math. Soc. vol. 67 (1961) pp. 607-608.

The University of British Columbia 